\title{
Similitude Analysis of Concrete Scaled Bridge Columns for Quasi-Static and Free Vibration Testing
}

\author{
Ali M. Syed ${ }^{1}$, Mohammad Javed ${ }^{1}$, Bashir Alam ${ }^{1}$ \\ ${ }^{1}$ Faculty member, Department of Civil Engineering, University of Engineering \& Technology Peshawar, \\ Peshawar 25120, Pakistan
}

\begin{abstract}
Structural testing in laboratory often requires scaled models for testing. Bridges generally have tall columns and heavy loads, therefore require scaling down the model columns to suit laboratory facilities, cost and schedule constraints. However, scaling is not a straight forward procedure, it requires fulfillment of requirements derived from similitude analysis. While undertaking similitude studies, some parameters can be chosen by the researcher whereas other parameters would then be constrained. In this paper a detailed account of similitude analysis for four scaled bridge columns is presented in which reverse quasi-static cyclic testing is done along with free vibration tests. The similitude requirements for static and dynamic tests are different and discussed here in detail to explain how best various requirements are fulfilled.
\end{abstract}

Key Words: Similitude, bridges, quasi-static cyclic testing, free vibration testing.

\section{Introduction}

Single column bridges are important class of bridge sub-structure system and they are described in bridge design specifications [1]. In developing countries due to quality control issues and lack of standard construction equipment, often low strength concrete bridge columns are seen [2], [3]. In seismic events lateral motion of bridges due to ground shaking causes the cracking and yielding in the plastic hinge zone of columns, and results in energy dissipation through hysteresis [4], [5]. Four scaled bridge columns were prepared and tested in laboratories of Earthquake Engineering Center. The scaled reinforced concrete (RC) bridge columns had solid circular section. The four scaled columns were primarily tested for reverse quasi-static cyclic testing, however a portion of testing comprised of free vibration studies also [2]. For lab testing several issues are to be resolved before any testing is undertaken. Usually it is rare to construct full scale test models due to their size, cost, time and limitation of laboratory equipment [6]. This paper discusses in detail similitude analysis required for laboratory testing of scaled bridge column while considering various limitations of laboratory equipment and other facilities. The typical setup of laboratory is shown in Figure 1.

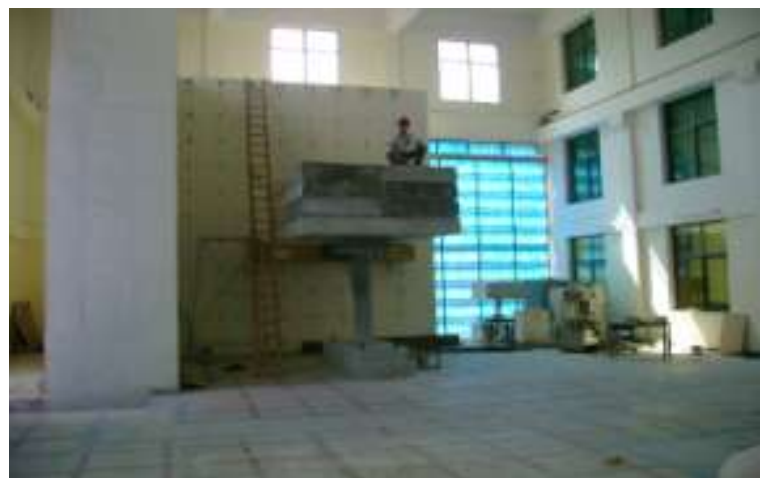

Figure 1: A typical laboratory setup.

\section{Formulation Of Test Methodology Forming Basis Of Similitude Analysis}

It is very important to know and understand the specifications, capacity and limitation of test equipment and laboratory facilities. In this study various laboratory equipment were studied in detail by constructing few trial test columns. In light of limitation of lab equipment and from carrying out the testing on trial columns, it was decided to carryout Quasi-Static Cyclic testing on RC bridge columns for the study on energy dissipation capacity. It was decided that scale factor for geometric scaling would be taken as 1:4 as this would result in a reasonable and manageable size test columns. Here it is worth mentioning that selection of scale for the model is dependent on many factors which include the laboratory facilities and limitation of test equipment [7]. An important decision was made with regard to dead mass simulation, and it was decided to physically fabricate the actual required mass for the scaled models. The purpose was to avoid boundary 
condition problem. Secondly it was decided to carryout Hybrid Quasi-Static Cyclic Testing that involved not only conventional way of performing the quasi-static cyclic testing but also involved Free Vibration Testing of the RC model column to study the dynamic behavior of the model test column under test load. It was believed that the dynamic data obtained from the ambient vibrations of the test column would provide data that would be used in determining the actual dynamic characteristics of the test columns. Similarly dynamic behavior of test columns would be studied at various levels of inelasticity which would result from quasi-static cyclic testing of columns under various levels of lateral drift demand. It was believed that such dynamic data would be extremely helpful in establishing actual behavior of RC columns at various states that would include knowing the actual parameters such as natural period of vibration, damping of the system, and actual effective stiffness, at various stages of increasing damage.

\section{Similitude Analysis}

After careful study of the requirements of dynamic and quasi-static testing, study of operational requirements of the laboratory equipment, physical limitations of the laboratory space, precision and limitations of sensors to be used, limitations of the data acquisition system and financial constraints, various important decisions were made that are described in detail in following sections.

\section{Scale Factor Related To Geometry}

Various factors related to Dimension of "Length" are discussed here.

\subsection{Scale Factor for Length}

Size of a specimen is one of the most important parameter from various prospects, therefore the scale factor for length $\lambda_{l}$ was of significance and needed due care in selection. The scale factor for length can make the model very large if a smaller value of scale factor is selected which can result in cost implications, limitation of lifting devices and limitation of loading devices available in the lab, as well as space limitation. And if a very large value of this scale factor is selected, it can result in difficulties associated with fabrication, tolerances, difficulty in getting small diameter rebar having deformations etc. [7].

The choice of scale factor also depends on type of testing, if elastic studies are to be done then significantly small models can be prepared but for inelastic studies the strength models would require making relatively larger models. In bridge studies the range for strength models can vary between $\frac{1}{20}$ to $\frac{1}{4}$ [6]. The length is one of the basic independent dimensions used in dimensional analysis and it is related to various parameters in fulfilling the similitude requirements, and the effect of length goes beyond the affects of physical and administrative limitations. This factor affects other characteristics of materials and structural parameters such as specific weight, concentrated loads, moments, shear force and acceleration requirements. The scale factor for length (geometry) was pre-selected to be $\lambda_{l}=4.0$. This factor was considered to be the most suitable to result in a reasonable size specimen that could optimally cater for issues discussed in preceding sections. Thus from above discussion we have:

$$
\lambda_{l}=\frac{l_{p}}{l_{m}}=4.0
$$

Where: $\quad \lambda_{l}$ is the scale factor related to linear geometric terms;

$l_{p}$ is the length related parameter in prototype;

$l_{m}$ is the length related parameter in model.

\subsection{Scale Factor for Area}

In light of above, as the area is derived as a result of multiplication of two lengths, therefore the scale factor for area of the bridge column is described below:

$$
\lambda_{A}=\frac{A_{p}}{A_{m}} \rightarrow \lambda_{A}=\frac{l_{p}{ }^{2}}{l_{m}{ }^{2}} \rightarrow \lambda_{A}=\lambda_{l}{ }^{2} \rightarrow \lambda_{A}=16.0
$$

Where: $A_{p}$ and $A_{m}$ are factor related to area terms in prototype and model respectively;

$\lambda_{A}$ is the scale factor for area. 


\subsection{Scale Factor for Moment of Inertia}

The scale factor for moment of inertia is calculated from dimensional analysis to fulfill the similitude requirements, is give below:

$$
\lambda_{I}=\frac{l_{p}{ }^{4}}{l_{m}{ }^{4}} \rightarrow \lambda_{I}=\lambda_{l}{ }^{4} \rightarrow \lambda_{I}=64.0
$$

Where: $\lambda_{I}$ is the scale factor related to moment of inertia.

\subsection{Scale Factor for Linear Displacements}

The scale factor for linear displacements in prototype and model will have the same basic scale factor as that of length determined from dimensional analysis, to meet the requirement of similitude and is described below:

$$
\lambda_{D}=\frac{l_{p}}{l_{m}} \rightarrow \lambda_{D}=\lambda_{l} \rightarrow \lambda_{D}=4.0
$$

Where: $\lambda_{D}$ is the scale factor related to linear displacements.

\subsection{Scale Factor for Angular Displacements}

From dimensional analysis it is calculated that the scale factor for angular displacement will be unity. Thus the rotational value of a quantity in a model does not require modification as the scale factor is unity and angular quantities in model are equal to that in prototype. This is described below:

$$
\lambda_{\theta}=\frac{\frac{s_{p}}{r_{p}}}{\frac{s_{m}}{r_{m}}} \rightarrow \lambda_{\theta}=\frac{\frac{l_{p}}{l_{p}}}{\frac{l_{m}}{l_{m}}} \rightarrow \lambda_{\theta}=1.0
$$

Where: $\lambda_{\theta}$ is the scale factor related to rotational displacements;

$s_{p}$ is the distance traversed in an arc by a prototype;

$S_{m}$ is the distance traversed in an arc by a model;

$r_{p}$ is the radial distance in a prototype;

$$
r_{m} \text { is the radial distance in a model. }
$$

\section{Scale Factors Related To Material Properties}

It was decided to conduct quasi-static cyclic testing on RC columns which would push the columns into inelastic range in the region of plastic hinge. To avoid scaling of mechanical properties of concrete and reinforcing steel, it was decided to use same material characteristic in model as that of prototype, as modeling mechanical properties of rebar is rather difficult especially when dealing with inelastic material response. Using the same material properties eliminates many complications. Further the similitude rules do not satisfy inelastic behavior and are very complex, therefore [8] suggests using same prototype material in model and hold the same stress-strain rates.

\subsection{Scale Factor for Modulus of Elasticity}

In light of preceding section, it was established that same materials would be used thus the modulus of elasticity of reinforcing steel and concrete would also be same. Therefore scale factor for modulus of elasticity would be as under:

$$
\lambda_{E}=\frac{E_{p}}{E_{m}}=1.0
$$

Where: $\quad \lambda_{E}$ is the scale factor related to modulus of elasticity.

\subsection{Scale Factor for Stress}

Since it was pre-decided that same materials were to be used in model, as that of prototype therefore this leads to conclusion that the scale factor for stress would be unity and is described below: 


$$
\begin{gathered}
\operatorname{Stress}(\sigma)=\frac{\text { load }}{\text { area }} \rightarrow \sigma=\frac{F}{L^{2}} \rightarrow \sigma=E \rightarrow \lambda_{\sigma}=\frac{\sigma_{p}}{\sigma_{m}} \\
\lambda_{\sigma}=1.0
\end{gathered}
$$

Where: $\lambda_{\sigma}$ is the scale factor related to modulus of elasticity.

\subsection{Scale Factor for Specific Weight (column only)}

The RC column under test was made of two main components, one was column itself and second part was the dead mass supported on column top. Here in this section only the RC column is discussed. To fulfill the requirements of similitude on the basis of dimensional analysis carried out for specific weight of the material, result is presented below:

$$
\lambda_{\gamma}=\lambda_{\rho}=\frac{\lambda_{E}}{\lambda_{l}} ; \lambda_{E}=1.0 ; \lambda_{l}=4.0 \rightarrow\left(\lambda_{\gamma}\right)_{\text {required }}=\frac{1}{4}
$$

Where: $\lambda_{\gamma}$ is the scale factor related to specific weight of material.

The Eq. 8 is the scale factor for specific weight in the case when no dynamic excitation is involved which implies to quasi-static testing only.

However, for the case of dynamic excitation the requirement for scale factor related to specific weight would be as follows:

$$
\lambda_{\gamma}=\frac{\lambda_{E}}{\lambda_{l} \lambda_{a}} \rightarrow \lambda_{E}=1.0 ; \lambda_{l}=4.0 ; \rightarrow\left(\lambda_{\gamma}\right)_{\text {required }}=\frac{1}{4 \cdot \lambda_{a}}
$$

Where: $\quad \lambda_{a}$ is the scale factor related to acceleration.

Here the value of $\lambda_{a}$ needs to be preselected, a natural choice would be to take its value as unity. This would result in:

$$
\left(\lambda_{\gamma}\right)_{\text {required }}=\frac{1}{4}
$$

However, as described in preceding sections it was pointed out that similar materials are used thus the provided specific weight for the model is:

$$
\left(\lambda_{\gamma}\right)_{\text {provided }}=1.0
$$

This study involved predominately quasi-static cyclic testing which does not involve the excitation of mass and the required specific weight is described in Eq.8. Whereas provided specific weight is described in Eq.11. The specific weight requirement here in quasi-static test is only related to produce correct stresses in column due to weight. It would be shown latter that this deviation from requirement of similitude is harmless as column mass is hardly $1.8 \%$ of the total mass.

\subsection{Scale Factor for Poisson's Ratio}

Since Poisson's ratio is a dimensionless number therefore the scale factor for it is always unity.

$$
\lambda_{v}=1.0
$$

\subsection{Scale Factor for Strain}

Strains are also dimensionless and therefore the scaling factor for strain is also always unity.

$$
\lambda_{\varepsilon}=1.0
$$

\section{Scale Factors Related To Loading}

Various forms of loading exists which need to be scaled appropriately, these are discussed here.

\subsection{Scale Factor for Concentrated Load}

The concentrated load when scaled need to fulfill the requirements of similitude. From the dimensional analysis, the scale factor for concentrated load is calculated as under.

$$
\lambda_{Q}=\lambda_{E} \cdot \lambda_{l}^{2} ; \lambda_{E}=1.0 ; \lambda_{l}=4.0 \rightarrow \lambda_{Q}=16.0
$$


Where: $\lambda_{Q}$ is the scale factor related to concentrated force.

\subsection{Scale Factor for Shear Force}

From the dimensional analysis the scale factor for shear force is calculated as follows:

$$
\lambda_{V}=\lambda_{E} \cdot \lambda_{l}^{2} ; \lambda_{E}=1.0 ; \lambda_{l}=4.0 \rightarrow \lambda_{V}=16.0
$$

Where: $\lambda_{V}$ is the scale factor related to shear force.

\subsection{Scale Factor for Moment}

The scale factor for moment is calculated from dimensional analysis as follows:

$$
\lambda_{M}=\lambda_{E} \cdot \lambda_{l}{ }^{3} ; \lambda_{E}=1.0 ; \lambda_{l}=4.0 \rightarrow \lambda_{M}=64.0
$$

Where: $\lambda_{M}$ is the scale factor related to bending moment.

\section{Scale Factors Related To Dynamic Characteristics}

This study on RC bridge piers was carried out using quasi-static testing. However a hybrid approach was used during the cyclic testing as suggested by Prof. S. A. Sheikh [9] which employed the concept of Ambient Vibration Testing also called free vibration testing [10] at various stages of quasi-static cyclic testing to study the dynamic characteristics of RC model columns as a mean to identify their behavior especially in nonlinear material range. Related scale factors are discussed below.

\subsection{Scale Factor for Mass (lumped on column top)}

In this study the test column was analogous to an inverted pendulum with mass lumped on top of the $\mathrm{RC}$ column. The lumped mass represented the mass of the super-structure in a bridge system. From dimensional analysis carried out for the lumped mass, the scale factor is provided below:

$$
\lambda_{m}=\lambda_{\rho} \cdot \lambda_{l}^{3} ;\left(\lambda_{\rho}\right)_{\text {required }}=\frac{1}{4} ; \lambda_{l}=4.0 \rightarrow\left(\lambda_{m}\right)_{\text {required }}=16.0
$$

Where: $\lambda_{m}$ is the scale factor related to mass;

$\lambda_{\rho}$ is the scale factor related to mass density, the scale factor is same as that for specific weight calculated in Eq.8.

From Eq. 17 the mass required for the model is calculated below:

$$
\left(\lambda_{m}\right)_{\text {required }}=16 \rightarrow \frac{M_{p}}{M_{m}}=16 \rightarrow M_{m}=\frac{M_{p}}{16} \rightarrow M_{m}=\frac{\gamma_{p} \cdot V_{p}}{16}
$$

Where: $M_{p}$ is the mass of prototype structure;

$M_{m}$ is the mass in model;

$\gamma_{p}$ is the specific weight of prototype material.

It is important to note that the provided scale factor for the mass is 16.0 Thus the mass similitude requirements for the lumped mass on column top is fulfilled and the column top mass is $98.2 \%$ of the total mass of the system.

\subsection{Scale Factor for Acceleration}

It is important to consider the scale factor for the acceleration as this would later be used in calculating the scale factor for natural period of the RC column system. From dimensional analysis the scale factor for the acceleration is described in Eq.9. It was pointed out there that this factor was preselected as unity.

$$
\lambda_{a}=1.0
$$

It is important to mention here that this formed the basis to calculate the scale factor for specific mass which then came out to be $\left(\lambda_{\gamma}\right)_{\text {required }}=\frac{1}{4}$ for the case of column only as stated in Eq.10. However, for the top mass the scale factor for the net mass was calculated to be 16 in Eq. 17. 
The acceleration due to gravity is constant and was taken unity in model and prototype; however it is possible to have tests in which similitude would require to scale acceleration due to gravity which would require use of equipment called centrifuge. For this study the scale factor for acceleration due to gravity is given below:

$$
\lambda_{g}=1.0
$$

Where: $\lambda_{g}$ is the scale factor related to acceleration due to gravity.

\subsection{Scale Factor for Natural Period}

From the dimensional analysis for natural period of the column system, results are presented as under:

$$
\lambda_{t}=\sqrt{\frac{\lambda_{l}}{\lambda_{a}}} ; \lambda_{l}=4 ; \lambda_{a}=1 \rightarrow \lambda_{t}=2.0
$$

Where: $\lambda_{t}$ is the scale factor related to time period.

Here it is very important to recall that Eq.21 is based on the premise that specific mass of the system is $\left(\lambda_{\gamma}\right)_{\text {required }}=\frac{1}{4}$. However, this assumption holds only for the top mass and not for the column itself, but this deviation did not cause any harm to the modeling and its associated results because of the fact that predominant mass contribution from the column top mass amounted more than $98.2 \%$.

\subsection{Scale Factor for Damping Ratio}

The damping ratio is a dimensionless quantity the scale factor is thus unity and described below:

$$
\lambda_{\xi}=1.0
$$

Where: $\lambda_{\xi}$ is the scale factor related to damping ratio.

\subsection{Scale Factor for Energy Dissipated}

The scale factor for energy dissipated by the RC column in inelastic range would be estimated by following equation:

$$
\begin{gathered}
e=f \cdot d \rightarrow e=\left(E L^{2}\right) \cdot L \rightarrow e=E \cdot L^{3} \\
\lambda_{e}=\lambda_{E} \cdot \lambda_{l}^{3}
\end{gathered}
$$

Where: $\lambda_{e}$ is the scale related to energy dissipated;

$e$ is the energy dissipated;

$f$ is the force acting;

$d$ is the displacement due to application of force;

$E$ is the modulus of elasticity;

$L$ is the dimension of length.

\section{Discussions On Results}

Scale factors are presented in tabular form in Error! Reference source not found. 1 in similar fashion as that provided in a $\mathrm{PhD}$ dissertation [11] for comparing the required scale factors verses the provided scale factors.

In this study the preselected scaling factors were related to length, material and acceleration as described in preceding sections. From above $\lambda_{l}=4.0$; is preselected scale factor for length, and $\lambda_{E}=1.0$ is preselected scale factor for modulus of elasticity which refers to same material. It is thus evident that same acceleration is used as scale factor is unity. All the other scale factors are then accordingly calculated by dimensional analysis to fulfill the similitude requirements. 


Table 1: Summary of scale factors used for test columns.
\begin{tabular}{|l|c|c|}
\hline \multirow{2}{*}{ Item } & \multicolumn{2}{|c|}{ Scale Factor } \\
\cline { 2 - 3 } & Required & Provided \\
\hline Length, $l$ & 4.0 & 4.0 \\
\hline Area, $A$ & 16.0 & 16.0 \\
\hline Moment of inertia, $I$ & 64.0 & 64.0 \\
\hline Linear displacement, $D$ & 4.0 & 4.0 \\
\hline Angular displacement, $\Theta$ & 1.0 & 1.0 \\
\hline Modulus of elasticity, $E$ & 1.0 & 1.0 \\
\hline Stress, $\sigma$ & 1.0 & 1.0 \\
\hline Specific mass for column only-static case, $\rho$ & 0.25 & 1.0 \\
\hline Poisson's Ratio, $V$ & 1.0 & 1.0 \\
\hline Strain, 8 & 1.0 & 1.0 \\
\hline Concentrated load, $\rho$ & 16.0 & 16.0 \\
\hline Shear force, $V$ & 16.0 & 16.0 \\
\hline Moment, M & 64.0 & 64.0 \\
\hline Mass on column top, $m$ & 16.0 & 16.0 \\
\hline Gravitational acceleration, $g$ & 1.0 & 1.0 \\
\hline Energy, $。$ & 64.0 & 64.0 \\
\hline
\end{tabular}

It is pointed out here that all the similitude requirements are met, except a minor deviation to fulfill the requirement of specific mass for RC column only. The total mass of the whole test specimen was 19.6 ton force (tf), which comprised of the top mass and mass of column itself. Similitude requirement was met for top mass that amounts to $19.2 \mathrm{tf}$ which makes $98.3 \%$ of the total required mass. However, since the required scale factor for the specific mass was 0.25 and provided scale factor was 1 for the RC column case only; this deviation resulted in an error of $1.3 \%$ in total mass of the test object. It is important to realize that the mass of column was a distributed mass over the entire height which makes the share of column mass even less, thus further reducing the deviation from $1.3 \%$ in dynamic response measurements such as for finding the time period of the system. However the contribution of this $1.3 \%$ deviation in static response that is related to stresses is also negligible since this offset would just introduce an estimated error of $\pm 0.034 \mathrm{MPa}$ only, which causes an approximate error of $\pm 0.25 \%$ in stress measurements.

\section{Conclusions}

This paper shows that in order to get reliable results from laboratory testing of scaled test specimens, which are concrete column in this case, requires to undertake detailed similitude analysis. It is seen in this study that the similitude requirements for static and dynamic testing are different from each other. This study further shows that design of experiment depends upon choosing appropriate scale factors. The scaling factors have to be such that various types of constraints are met with. Generally, the constraints are limitation of laboratory facilities, cost, fabrication complications, schedule etc. Some of the scale factors can be chosen by the experimentalist to suit its conditions, whereas scale factors for other parameters would then be calculated which would be coupled with few of the pre-selected scale factors.

\section{Acknowledgment}

The authors wish to thank HEC Islamabad for their support for PhD of first author.

[1] AASHTO LRFD, 2007, "AASHTO LRFD, Bridge Design Specification", $4^{\text {th }}$ edition. Washington D.C. (USA): The American Association of State Highway Officials"

[2] Syed A. M. 2009, "Study of Energy Dissipation Capacity of RC Bridge Columns under Seismic Demand" Ph.D. dissertation. NWFP University of Engineering and Technology Peshawar, Pakistan

[3] Syed A. M., Khan N. A., Rahman S., Reinhorn A., M. 2011, "A Survey of Damages to Bridges in Pakistan after a Major Earthquake of October 8, 2005". Earthquake Spectra, 27: 947-970.

[4] Kawashima, K. (2006). Seismic Design, Isolation and Retrofit of Bridge. Tokyo: Department of Civil Engineering Tokyo Institute of Technology, Japan.

[5] Poljansek, K., Perus, I., \& Fajfar, P, 2009, "Hysteretic energy dissipation capacity and the cyclic to monotonic drift ratio for rectangular RC columns in flexure", Earthquake Engineering and Structural Dynamics, 38, 907-928.

[6] Harris, H. G., \& Sabnis, G. M. (1999). Structural Modeling and Experimental Techniques. Florida: CRC Press LLC.

[7] Mander, J. B., Waheed, S. M., Chaudhary, M. T., \& Chen, S. S. (1993). Seismic Performance of Shear-Critical Reinforced Concrete Bridge Piers. Buffalo, NY: National Center for Earthquake Engineering Research (NCEER), SUNY.

[8] Reinhorn, A. M. (2008, Dec 23). Lecture 2 - Modeling of Structures and Similitude. Retrieved Dec 23, 2008, from CIE616 EXPERIMENTAL METHODS IN STRUCTURAL ENG.: http://civil.eng.buffalo.edu/CIE616/LECTURES/Lecture\%202\%20$\% 20$ Modeling $\% 20$ and $\% 20$ Scaling/Slides $\% 202 \% 20 \% 20$ Modeling\%20and\%20Scaling.pdf

[9] Sheikh, S. A. (2008, January 9). Visit to Earthquake Engineering Center NWFP UET Peshawar Pakistan. (A. M. Syed, Interviewer)

[10] Chopra, A. K. (2001). Free Vibration Tests. In A. K. Chopra, Dynamics of Structures Theory and Applications to Earthquake Engineering (2nd ed., pp. 54-55). New Jersy, USA: Prentice-Hall Inc.

[11] Bracci, J. M. (1992). Experimental and Analytical Study of Seismic Damage and Retrofit of Lightly Reinforced Concrete Structu res in Low Seismicity Zones. Buffalo: State University of New York. 